\title{
Effect of graphene grains size on the microwave electromagnetic shielding effectiveness of graphene/polymer multilayers
}

\author{
Polina P. Kuzhir ${ }^{\mathrm{a}}$, Alesia G. Paddubskaya ${ }^{\mathrm{b}, ~}$, Nadzeya I. Volynets ${ }^{\mathrm{a}}$, Konstantin G. \\ Batrakov $^{\mathrm{a}}$, Sergey A. Maksimenko ${ }^{\mathrm{a}}$, Elena N. Golubevac ${ }^{\mathrm{c}}$, Gintaras Valusis ${ }^{\mathrm{b}}$, Tommi Kaplas ${ }^{\mathrm{d}}$, \\ Nicolas Reckinger ${ }^{\mathrm{e}}$, Michael Lobet ${ }^{\mathrm{e}}$, Philippe Lambin ${ }^{\mathrm{e}}$ \\ ${ }^{a}$ Research Institute for Nuclear Problems, Belarusian State University, Bobruiskaya str. 11, Minsk, Belarus, 220030 \\ ${ }^{\mathrm{b}}$ Center for Physical Sciences and Technology, A. Gostauto 11, Vilnius, Lithuania, LT-01108 \\ ${ }^{c}$ Belarusian State University, Nezavisimosti avenue 4, Minsk, Belarus, 220030 \\ ${ }^{\mathrm{d}}$ Institute of Photonics, University of Eastern Finland, P. O. Box 111, Joensuu, Finland, FI-80101 \\ ${ }^{e}$ Physics Department, Universite de Namur, 61 Rue de Bruxelles, Namur, Belgium, B-5000
}

\begin{abstract}
The influence of CVD graphene grain size on the electromagnetic (EM) shielding performance of graphene/polymethyl methacrylate (PMMA) multilayers in $K_{a}$-band was studied both experimentally and theoretically. We found that increasing the average graphene grain size from 20 to 400 microns does not change the EM properties of heterostructures consisting of graphene layers sandwiched between sub-micron thick PMMA spacers. The independence of EM interference shielding effectiveness on the graphene grain size between 20 to 400 microns allows one to use cheaper (or more convenient regimes of CVD) graphene samples with low crystallinity and small grain size in the development of new graphene-based passive electromagnetic devices operated at high frequencies.
\end{abstract}

Keywords: microwaves, electromagnetic shielding, graphene grain size, heterostructure, CVD graphene.

*Alesia G. Paddubskaya, paddubskaya@gmail.com

\section{Introduction}

The quality of wafer-size graphene is still remaining the major bottleneck in the fabrication and mass production of novel photonic and optoelectronic devices relying on unique properties of Dirac electrons. ${ }^{1-4}$ This is because the presence of defects, grain boundaries, multiple domains, impurities and other irregularities in the graphene sheet inevitably affects the carriers mobility, provoking thereby a deterioration of the electronic and optical properties. Among the variety of graphene fabrication techniques, such as mechanical exfoliation of graphite, ${ }^{5}$ sublimation of epitaxial $\mathrm{SiC}^{6}$ and catalyct-assisted chemical vapor deposition (CVD), ${ }^{2,7-10}$ the CVD is the most promising route for scalable graphene fabrication. However, the polycrystalline character of CVD graphene implies electron scattering on boundaries between adjacent crystallites that strongly influence the transport, ${ }^{11}$ thermal and electrical properties, as well as the contact resistance of the 
graphene/metal interface, thus reducing the performance of high-frequency graphene electronic devices. ${ }^{12,13}$ In particular, it has been shown that grain boundaries result in prominent weak localization effects indicative of inter-valley charge carrier scattering that impedes electrical transport in CVD graphene. ${ }^{14}$ This makes it difficult to employ CVD graphene in practical electronic components and devices capable to generate, detect and process electromagnetic (EM) signals.

On the other hand, one may expect that the ability of graphene for absorbing electromagnetic radiations at high frequency be less sensitive (in comparison with transport properties) to the grain size. Such an intuitive conclusion is based on the fact that under EM radiations with wavelength of about $1 \mathrm{~cm}$ the micron-sized graphene grains are coupled not only electrically, but also electromagnetically.

Recently, we demonstrated that graphene/polymer (polymethyl methacrylate (PMMA)) multilayers can provide an efficient far-field shielding against microwave radiations, allowing one to achieve up to $50 \%$ absorption of the incident radiation. ${ }^{15}$ The goal of this paper is to investigate both experimentally and theoretically the influence of CVD graphene grain size on the electromagnetic shielding performance of such sandwich structures. In a broader sense, we aim at revealing the effects of the CVD graphene quality on the electromagnetic properties of passive electromagnetic devices.

\section{Modelling}

The Rigorous Coupled-Wave Analysis (RCWA) method ${ }^{16}$ has been applied to theoretically explore the role of graphene grain sizes in the total high-frequency electromagnetic shielding performance of graphene-based multilayers. This method is well adapted to plane-stratified systems with a periodic variation of dielectric permittivity in the lateral directions. ${ }^{17,18}$ 
The frequency used in the calculations was $30 \mathrm{GHz}(1 \mathrm{~cm}$ wavelength in vacuum), the PMMA/graphene multilayer was treated as a stack of dielectric slabs (PMMA spacers, 100 nm thick, permittivity at the working frequency of $2.6^{18}$ ), alternating with graphene layers and deposited on a $\mathrm{SiO}_{2}$ substrate with permittivity $3.7 .{ }^{18}$ Each graphene layer was treated as ultra-thin conducting medium (thickness $d_{\text {graph }}=0.34 \mathrm{~nm}$ ) with complex bulk dielectric function $\epsilon(\omega)=2.5+i \frac{\sigma_{2 D}}{\epsilon_{0} \omega d_{\text {graph }}}$. In that expression, $\sigma_{2 D}$ is the sheet conductivity of graphene, which for GHz frequency is dominated by intra-band transitions. ${ }^{19,20}$ For a given sample, the sheet conductivity of graphene varies only weakly with frequency up to the THz regime. ${ }^{21,22}$ We took the value $\sigma_{2 D} / \epsilon_{0} c=0.37$ used in our previous works (Ref. 17)

The electromagnetic modes were calculated in each layer of a graphene/PMMA heterostructure and then analytically propagated through the system by applying appropriate boundary conditions at each interface. Transmission $(T)$ and reflection $(R)$ coefficients were retrieved from the Poynting vector computed in both incidence and emergence media. The absorbance $(A)$ followed from $A=1-T-R$.

The polycrystalline character of CVD graphene introduces boundaries between adjacent grains. The width of those grain boundaries is estimated to be $3-5 \mathrm{~nm} .^{23}$ In the present simulations, random grains were created with average size of $20 \mu \mathrm{m}$ (Fig. 1 a) and $400 \mu \mathrm{m}$ (Fig. 1 b). The grain boundaries between adjacent grains were implemented with a width of approximately $5.5 \mathrm{~nm}$ and a dielectric permittivity of 1 . This stands for the worse possible electrical situation, since the grain boundaries are therefore not made of a conducting material anymore.

The modeling results for absorption, transmission and reflection provided by graphene/PMMA sandwiches consisting of graphene with different grain size are presented in Fig. 1c. The curves obtained for the two kinds of graphene can barely be distinguished, demonstrating that the electro- 

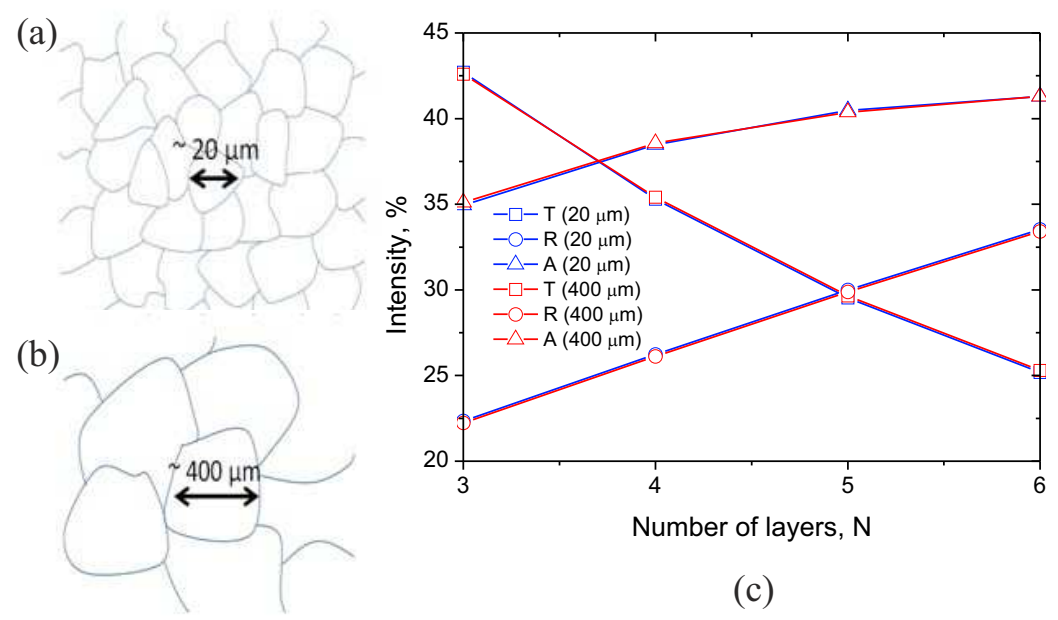

(c)

Fig 1 Graphene grain patchwork for small $(20 \mu \mathrm{m})$ (a) and large (400 $\mu \mathrm{m})$ (b) graphene grains drawn from experiment and transposed to RCWA calculations to investigate the effects of grain boundaries on the electromagnetic properties of a graphene/PMMA multilayer. The blue lines, $5.5 \mathrm{~nm}$ thick, have a dielectric permittivity of 1 , the rest has the permittivity of graphene. (c) Absorbance $(A)$, reflectance $(R)$ and transmittance $(T)$ of graphene/PMMA sandwich structures composed of $N$ graphene layers in case of graphene made of small (red lines) and large (blue lines) grains.

magnetic response is not sensitive to the grain size. In both case, the grain size are smaller than the incident wavelength of $\mathbf{1} \mathbf{~ c m}$ in vacuumm, which becomes $0.63 \mathbf{~ c m}$ (a factor of $1 / \sqrt{2.6}$ smaller) in the heterostructure and an effective medium theory can be used to describe the graphene dielectric permittivity with different grain size. As long as the total area occupied by the boundaries remains negligible compared to the total area occupied by the grains, the grain boundaries have no effects on the electromagnetic properties of graphene. Those results can be compared with those for a few percentages of small air holes in the graphene sheets that already proved not affecting the electromagnetic properties of graphene. ${ }^{17}$ 


\section{Experimental details}

\subsection{Samples fabrication and quality control}

On the experimental side, sandwich structures based on graphene with different grain size were produced. The graphene was synthesized by chemical vapor deposition (CVD) at atmospheric pressure. The copper foil (99.9\% purity; $50 \mu \mathrm{m}$ thick; $1 \mathrm{~cm}^{2}$ ) was first sonicated in acetone for 15 min, then in isopropanol for 15 min and finally deoxidized in acetic acid (99.5\% purity) at $35{ }^{\circ} \mathrm{C}$ for 10 min. Next, it was inserted into a quartz tube inside a hot-wall furnace whose temperature was set at $1050{ }^{\circ} \mathrm{C}$. In order to provide different size of grains the graphene (GRA) deposition was made in two regimes:

- Samples with small grains (about $20 \mu \mathrm{m}$ ) were produced as follows: (1) a 1-hour time annealing of the $\mathrm{Cu}$ foil under argon (500 sccm, $99.9 \%$ purity) and hydrogen (20 sccm, 99.9 $\%$ purity); (2) graphene growth by filling the chamber with $1 \mathrm{sccm}$ of diluted methane (5\% in argon) for 1 hour; (3) fast cooling of the sample in the same gas mixture.

- The "large grain size" samples were grown identically as for the small-grain samples with one difference in the $\mathrm{Cu}$ annealing step. For the first $30 \mathrm{~min}$, the annealing was in argon flow $(500 \mathrm{sccm})$, while during the following $30 \mathrm{~min}$, the annealing was with an argon/hydrogen mixture $(500 / 20 \mathrm{sccm})$. Thereby, during the first $30 \mathrm{~min}$ of the annealing, the copper foil surface was slightly oxidized thanks to residual impurities in the argon flow, ${ }^{24}$ the so-called oxidative annealing was applied. ${ }^{25,26}$

To determine the grain size, the synthesis of test samples was stopped before graphene covered the whole copper substrate. The morphology and structure of the graphene domains were con- 

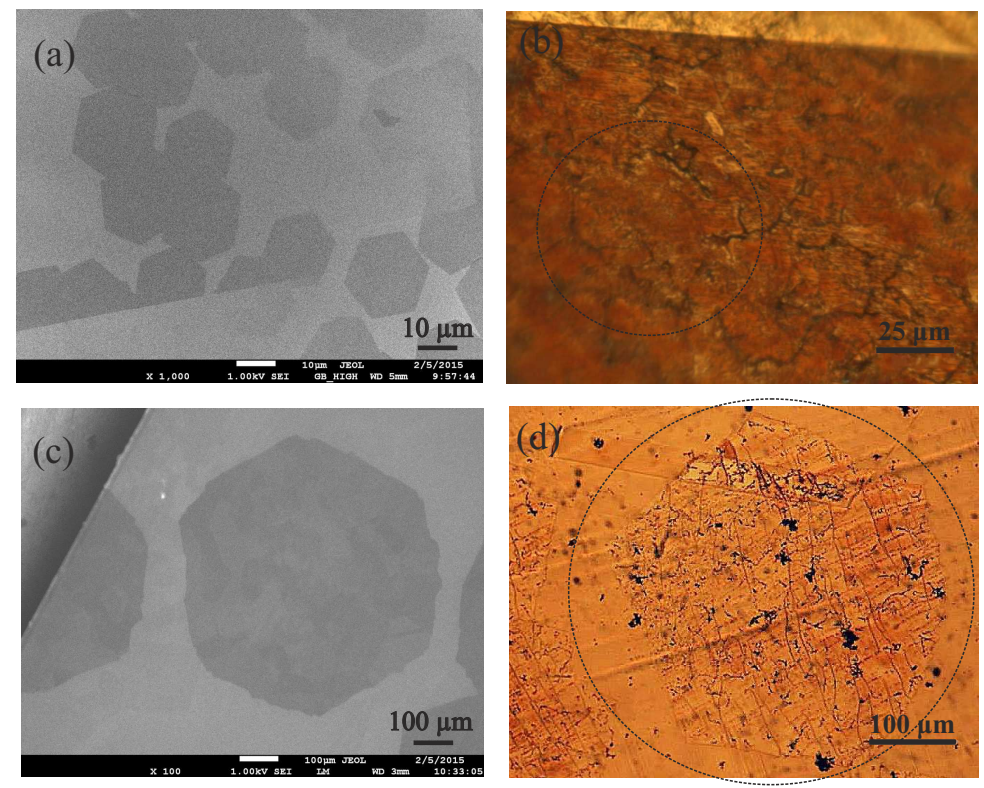

Fig 2 SEM images of graphene hexagonal domains corresponding to $20 \mu \mathrm{m}-\mathrm{GRA}$ (a) and $400 \mu \mathrm{m}-\mathrm{GRA}$ (c) samples, when the synthesis was stopped before graphene covered the whole copper substrate. (b), (d) optical images of graphene samples after chemical treatment obtained according to technique proposed in Ref. 27. The disks drawn with a thin dotted line in (b) and (d) indicate the graphene domains in case of small and large grain size samples, respectively

trolled by Field Emission Scanning Electron Microscope (FE-SEM, JEOL JSM-7500 F). As can be seen in Fig. 2 a and c, the graphene domains have a hexagonal shape with uniform color contrast and average lateral size around $20 \mu \mathrm{m}(20 \mu \mathrm{m}-\mathrm{GRA})$ and $400 \mu \mathrm{m}(400 \mu \mathrm{m}-\mathrm{GRA})$, respectively.

Additionally, after CVD deposition graphene grain boundaries were visualized by using a simple technique proposed by S. Yu et al.. ${ }^{27}$ basic permanganate solution was used as an oxidant and graphene grain boundaries were observed by means of optical microscopy (MI-1, Planar, Belarus, the measurements were done in the reflection mode with a with a $100 \times$ and $80 \times$ objectives). Fig. 2 b and d show typical images of graphene samples with domain size of $20 \mu \mathrm{m}$ and $400 \mu \mathrm{m}$, respectively. One can observe in Fig. 2 that images of the graphene grains provided by optical microscope and SEM give the same size of the graphene grains. 

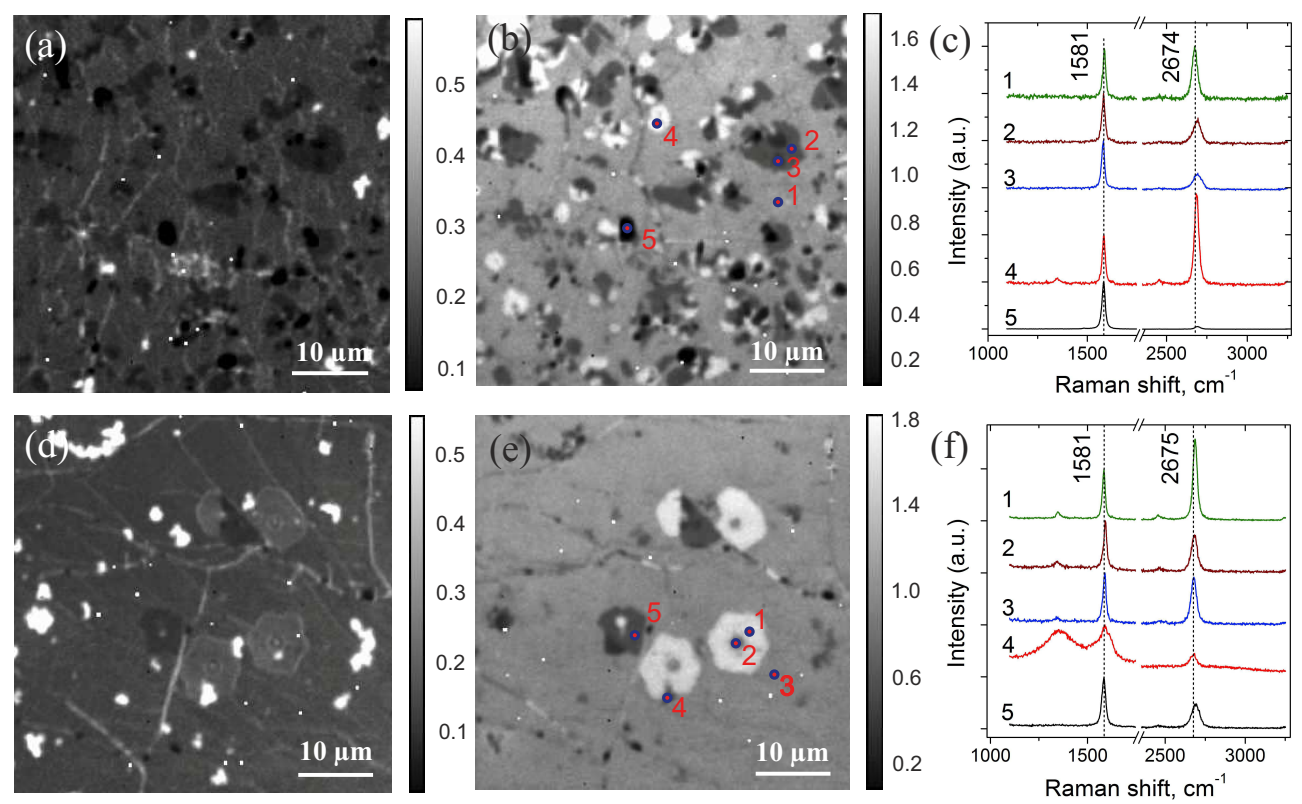

Fig 3 Raman maps of the intensity ratio of D- and G-bands over a $40 \times 40 \mu \mathrm{m}$ area of $20 \mu \mathrm{m}-\mathrm{GRA}$ (a) and 400 $\mu$ m-GRA (d), respectively. Raman maps of the intensity ratio of 2D- and G-bands over a $40 \times 40 \mu \mathrm{m}$ area of 20 $\mu \mathrm{m}$-GRA (b) and $400 \mu \mathrm{m}$-GRA (e), respectively. (c), (f) Raman spectra corresponding to the different regions marked with dots in (b) and (e). Spectra labelled 1 in (c) and 3 in (f) are typical of graphene monolayer. All spectra were normalized on the $\mathrm{G}$ band peak amplitude

Raman mapping was further used to analyze the quality and uniformity of the graphene sheet. For this purpose, graphene films were transferred onto $\mathrm{Si}$ wafers with a $300 \mathrm{~nm}$ thick $\mathrm{SiO}_{2}$ native oxide overlayer using the conventional "PMMA-mediated" technique. ${ }^{28}$ Measurements were performed using Raman spectrometer combined with a confocal microscope Nanonder High End (Tokyo Instruments) with a 600 lines/mm grating and $532 \mathrm{~nm}$ laser excitation. Spectra were collected using a $50 \times$ objective and at low power $(20 \mathrm{~mW})$ to reduce sample degradation (Fig. 3). From the analysis of Raman maps of the intensity ratio of $2 \mathrm{D}-\left(\sim 2675 \mathrm{~cm}^{-1}\right.$ with the typical FWHM value of $\left.35-40 \mathrm{~cm}^{-1}\right)$ and G- $\left(\sim 1581 \mathrm{~cm}^{-1}\right.$ with the typical FWHM value of 17-21 $\mathbf{c m}^{-1}$ ) bands, and Raman spectra from different points, it can be concluded that the largest part of both samples is graphene monolayer but small multilayer islands are also present on the surface. 
The sandwich-like graphene/PMMA structures were produced using the procedure described elsewhere. ${ }^{15}$ Briefly, the CVD graphene layer was spin coated with 100 nm-thick PMMA layer, after that, the $\mathrm{Cu}$ substrate was wet etched in ferric chloride and the obtained graphene / PMMA film was washed in distilled water and transferred onto a $0.53 \mathrm{~mm}$-thick fused silica substrate. In order to fabricate sandwich multilayers containing several graphene sheets separated by PMMA layers, the same procedure was repeated several times, each newlyproduced graphene/PMMA unit being deposited on top of the stack obtained at the previous step

It is worth noting that depending on the experimental conditions 4 -7-layered graphene/PMMA heterostructure reaches its the maximum possible $50 \%$ absorbance in free space and waveguide geometry. Thereby, measurements on samples with three and six graphene layers allow us to visualize the influence of the graphene crystalline size of the EM shielding performance when the number of layers is close to the optimal one.

\subsection{Electromagnetic response measurements}

Microwave measurements in the $K_{a}$-band (26-36 GHz) were made with a scalar network analyzer R2-408R (ELMIKA, Vilnius, Lithuania). The IEC 62431:2008(E) standard for reflectivity measurements of EM materials was used. The EM response of sample was measured as ratios of transmitted/input $\left(S_{21}\right)$ and reflected/input $\left(S_{11}\right)$ signals after insertion of the specimen into the transmission line (rectangular waveguide) perpendicular to wave propagation. The lateral dimensions of the samples fitted precisely the waveguide $7.2 \times 3.4 \mathrm{~mm}^{2}$ cross section. 


\section{Experimental results and discussion}

The measured results for graphene/polymer sandwich heterostructures $(20 \mu \mathrm{m}-\mathrm{GRA}$ and $400 \mu \mathrm{m}$ GRA) consisting of three and six graphene/PMMA units are presented in Fig. 4. As it was expected and proved by theory the EM properties of samples produced from the graphene of small and large grain size are the same for both three- and six-layered graphene/PMMA sandwiches. As it follows from Fig. $4 \mathrm{~b}$ the total EM shielding effectiveness $\left(S E=-20 \log \left(S_{21}\right)\right)$ of graphene/polymer sandwiches in $K_{a}$-band varies in the range of $9-7 \mathrm{~dB}$ and $13-11 \mathrm{~dB}$ for 3 and 6 graphene layers, respectively, which is in good agreement with the previously reported data. ${ }^{15,29,30}$ In particular, in Ref. 29, shielding effectiveness of graphene monolayer and multilayer structures has been investigated. It was shown that the SE of monolayer graphene is $2.27 \mathrm{~dB}$ and the average SE values of double- and triple-layer graphene were $4.13 \mathrm{~dB}$ and $6.91 \mathrm{~dB}$, respectively. In Ref. 30, it was demonstrate that the SE value of reduced graphene oxide (RGO) sheets interleaved between polyetherimide with the number of RGO layers to achieves 6.37 dB for double-PEI/RGO multilayer. It is important to note that each graphene/PMMA sandwich is less than 1 micron thick (the overall thickness of samples with 3 and 6 graphene/PMMA layers was $300-400 \mathrm{~nm}$ and $600-700 \mathrm{~nm}$, respectively), still the level of EM power attenuation is similar to that observed for conventional polymer composites loaded with 5-10 wt. \% of graphene nanoplatelets or carbon nanotubes ${ }^{31,32}$ but with overall thickness of fraction of mm, namely a factor of 1000 thicker.

Our experiments and theoretical findings in the $K_{a}$ microwave band correlate well with recent results on the electrical properties of graphene at THz frequencies. Specifically, Buron et al have shown that graphene layer with lateral size of up to hundreds of microns grown on single crystal 

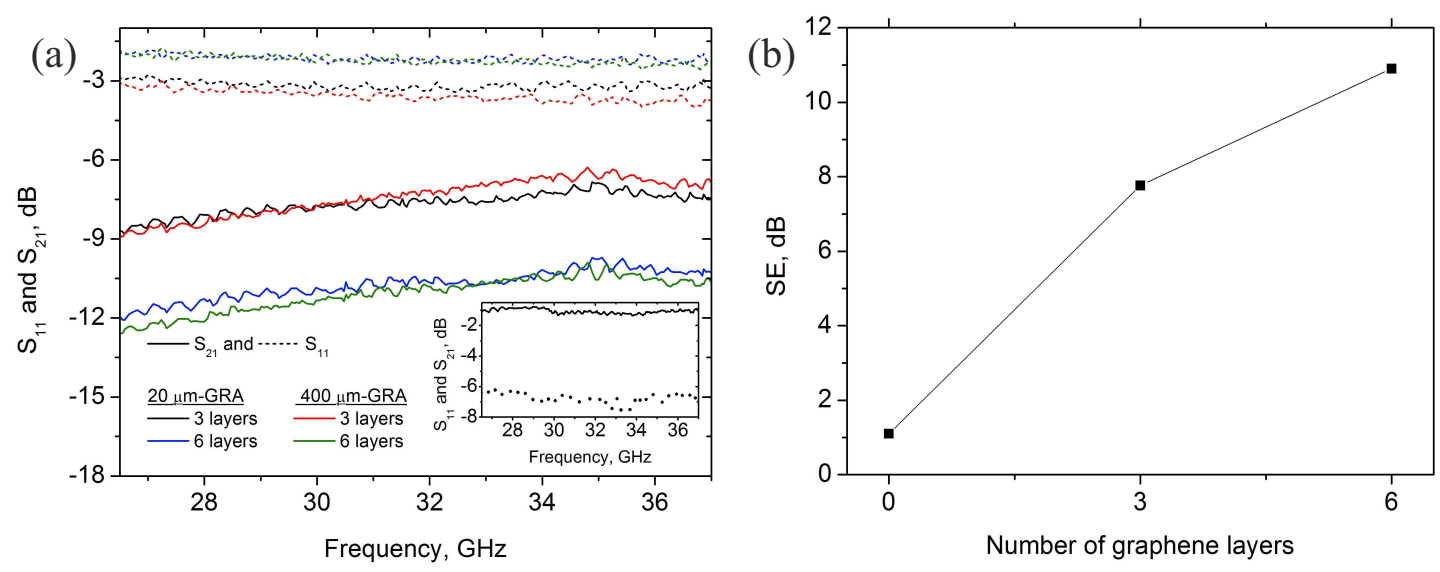

Fig 4 (a) Frequency dependence of $S_{11}$ and $S_{21}$ for graphene/polymer heterostructures on silica substrate consisting graphene with small and large grain size. Measured $S_{11}$ and $S_{21}$ for pure silica substrate ( $0.5 \mathrm{~mm}$ thickness) are shown inset. (b) The average SE of graphene/polymer heterostructures as function of the number of graphene layers at $30 \mathrm{GHz}$.

copper foil is electrically continuous at $1-15 \mathrm{THz} .^{22}$

\section{Conclusions}

The reported weak sensitivity of the results to the grain size of graphene together with high shielding effectiveness observed for graphene-based flexible thin multilayers opens an avenue for the development of a scalable protocol of cost-efficient production of ultra-light optically transparent EM shields. Shielding layers composed of inexpensive polycrystalline CVD graphene consisting of small graphene domains can be transferred to commercial applications.

\section{Acknowledgments}

The work was carried out within the framework of the FP7- FET Flagship 604391 Graphene and H2020 project 696656 Graphene Core1, supported partly by H2020 project 644076 CoExAN, FP7 project FP7-316633 POCAONTAS, the Academy of Finland grants \#287886 and \#298298, sponsor U.S. Air Force through CRDF Global Agreement grant AF20-15-61804-1. 


\section{References}

1 K. S. Novoselov, V. I. Falprimeko, L. Colombo, et al., "A roadmap for graphene," Nature 490, 192-200 (2012).

2 X. Li, W. Cai, J. An, et al., "Large-area synthesis of high-quality and uniform graphene films on copper foils," Science 324, 1312-1314 (2009).

3 S. Bae, H. Kim, Y. Lee, et al., "Roll-to-roll production of 30-inch graphene films for transparent electrodes," Nat Nano 5, 574-578 (2010).

4 W. Liu, H. Li, C. Xu, et al., "Synthesis of high-quality monolayer and bilayer graphene on copper using chemical vapor deposition," Carbon 49, 4122-4130 (2011).

5 K. S. Novoselov, A. K. Geim, S. V. Morozov, et al., "Electric field effect in atomically thin carbon films," Science 306, 666-669 (2004).

6 K. V. Emtsev, A. Bostwick, K. Horn, et al., "Towards wafer-size graphene layers by atmospheric pressure graphitization of silicon carbide," Nat Mater 8, 203-207 (2009).

7 A. Reina, X. Jia, J. Ho, et al., "Large area, few-layer graphene films on arbitrary substrates by chemical vapor deposition," Nano Lett. 9, 30-35 (2009).

8 W. Liu, B. L. Jackson, J. Zhu, et al., "Large scale pattern graphene electrode for high performance in transparent organic single crystal field-effect transistors," ACS Nano 4, 3927-3932 (2010).

9 Y. Wang, C. Miao, B. c. Huang, et al., "Scalable synthesis of graphene on patterned ni and transfer," IEEE Transactions on Electron Devices 57, 3472-3476 (2010).

10 A. Ismach, C. Druzgalski, S. Penwell, et al., "Direct chemical vapor deposition of graphene on dielectric surfaces," Nano Lett. 10, 1542-1548 (2010). 
11 L. Brown, E. B. Lochocki, J. Avila, et al., "Polycrystalline graphene with single crystalline electronic structure," Nano Lett. 14, 5706-5711 (2014).

12 I. Vlassiouk, S. Smirnov, I. Ivanov, et al., "Electrical and thermal conductivity of low temperature cvd graphene: the effect of disorder," Nanotechnology 22(27), 275716- (2011).

13 A. Hsu, H. Wang, K. K. Kim, et al., "Impact of graphene interface quality on contact resistance and rf device performance," IEEE Electron Device Letters 32, 1008-1010 (2011).

14 Q. Yu, L. A. Jauregui, W. Wu, et al., "Control and characterization of individual grains and grain boundaries in graphene grown by chemical vapour deposition," Nat Mater 10, 443-449 (2011).

15 K. Batrakov, P. Kuzhir, S. Maksimenko, et al., "Flexible transparent graphene/polymer multilayers for efficient electromagnetic field absorption," Scientific Reports 4, 7191- (2014).

16 M. G. Moharam and T. K. Gaylord, "Rigorous coupled-wave analysis of planar-grating diffraction," J. Opt. Soc. Am. 71(7), 811-818 (1981).

17 M. Lobet, N. Reckinger, L. Henrard, et al., "Robust electromagnetic absorption by graphene/polymer heterostructures," Nanotechnology 26(28), 285702- (2015).

18 P. Lambin, M. Lobet, K. Batrakov, et al., "Electrodynamics of graphene/polymer multilayers in the ghz frequency domain," in Fundamental and Applied Nano-Electromagnetics, 45-67, Springer Netherlands, Dordrecht (2016).

19 L. A. Falkovsky, "Optical properties of graphene," Journal of Physics: Conference Series 129(1), 012004- (2008).

20 F. H. L. Koppens, D. E. Chang, and F. J. Garca de Abajo, “Graphene plasmonics: A platform for strong lightmatter interactions," Nano Lett. 11, 3370-3377 (2011). 
21 N. Rouhi, D. Jain, S. Capdevila, et al., "Broadband conductivity of graphene from dc to thz," in Nanotechnology (IEEE-NANO), 2011 11th IEEE Conference on, 1205-1207 (2011).

22 J. D. Buron, F. Pizzocchero, B. S. Jessen, et al., "Electrically continuous graphene from single crystal copper verified by terahertz conductance spectroscopy and micro four-point probe," Nano Lett. 14, 6348-6355 (2014).

23 L. Tapaszto, P. Nemes-Incze, G. Dobrik, et al., "Mapping the electronic properties of individual graphene grain boundaries," Applied Physics Letters 100(5) (2012).

24 N. Reckinger, A. Felten, C. N. Santos, et al., "The influence of residual oxidizing impurities on the synthesis of graphene by atmospheric pressure chemical vapor deposition," Carbon 63, 84-91 (2013).

25 H. Zhou, W. J. Yu, L. Liu, et al., "Chemical vapour deposition growth of large single crystals of monolayer and bilayer graphene," Nature Communications 4, 2096- (2013).

26 L. Gan and Z. Luo, “Turning off hydrogen to realize seeded growth of subcentimeter singlecrystal graphene grains on copper," ACS Nano 7, 9480-9488 (2013).

27 S. U. Yu, Y. Cho, B. Park, et al., "Fast benchtop visualization of graphene grain boundaries using adhesive properties of defects," Chem. Commun. 49(48), 5474-5476 (2013).

28 X. Li, Y. Zhu, W. Cai, et al., "Transfer of large-area graphene films for high-performance transparent conductive electrodes," Nano Lett. 9, 4359-4363 (2009).

29 S. K. Hong, K. Y. Kim, T. Y. Kim, et al., "Electromagnetic interference shielding effectiveness of monolayer graphene," Nanotechnology 23(45), 455704 (2012).

30 S. Kim, J.-S. Oh, M.-G. Kim, et al., "Electromagnetic interference (emi) transparent shield- 
ing of reduced graphene oxide (rgo) interleaved structure fabricated by electrophoretic deposition,” ACS Appl. Mater. Interfaces 6, 17647-17653 (2014).

31 P. Kuzhir, A. Paddubskaya, D. Bychanok, et al., "Microwave probing of nanocarbon based epoxy resin composite films: Toward electromagnetic shielding," Thin Solid Films 519, 4114-4118 (2011).

32 F. Qin and C. Brosseau, "A review and analysis of microwave absorption in polymer composites filled with carbonaceous particles," Journal of Applied Physics 111(6) (2012).

Polina P. Kuzhir received her MD in theoretical physics from Belarusian State University in 1991 and $\mathrm{PhD}$ degree in theoretical and high energy physics, in 1996, from the Institute of Physics, Belarus Academy of Science, Belarus. She is currently a head of NanoElectroMagnetics lab at the Research Institute for Nuclear Problems, Belarus State University (BSU), Belarus. The general area of her scientific interest is fundamental and applied nanoelectromagnetics. She is actively involved in experimental research of electromagnetic response of nanocarbon composite materials in microwave and terahertz ranges. She participates in several FP7 and H2020 projects, including Graphene Flagship.

Alesia G. Paddubskaya received her MS degree in laser physics from Belarus State University, Minsk, Belarus, in 2010, where she is currently working toward the $\mathrm{PhD}$ degree. She is also a researcher at the Institute for Nuclear Problems, Belarus State University. Her current research interests include dielectric properties of composites with different forms of nanocarbon (singleand multiwall carbon nanotubes, carbon black, and onion-like carbon) over frequencies ranging from hertz to terahertz. 
Nadzeya I. Volynets received the M.S. degree in laser physics from Belarus State University (BSU), Minsk, Belarus, in 2013, and is currently working toward the Ph.D. degree at BSU. She is also currently a researcher with the Research Institute for Nuclear Problems, BSU. Her research interests include the investigation of electromagnetic (EM) properties of fluorinated graphene films and nanocarbon composite materials in microwave and terahertz ranges

Konstantin G. Batrakov is a senior researcher at INP BSU. He received his $\mathrm{PhD}$ in Theoretical Physics in 1993 from INP BSU. The results, obtained by Dr. Batrakov in the field of Free Electron Lasers have been used in the first lasing of Volume Free Electron Laser in INP BSU. His present research interest is graphene and CNTs applications as monomolecular light emitters in the $\mathrm{THz}$ frequency region (nano-scale free electron laser).

Sergey A. Maksimenko received the MS degree in physics of heat and mass transfer in 1976, the PhD degree in theoretical physics in 1988, both from Belarusian State University, Belarus, and the Doctor of Science degree in theoretical physics in 1996 from the Institute of Physics, Belarus National Academy of Science. Since 1992, he has been working as head of the Laboratory of electrodynamics of nonhomogeneous media at the Research Institute for Nuclear Problems, Belarus State University. He also teaches at the Belarus State University physical department. He has authored or coauthored more than 150 conference and journal papers. He is a chair of the international conference Fundamental and Applied Nanoelectromagnetics, Minsk, May 2012. He is SPIE Fellow and associate editor of the Journal of Nanophotonics. His current research interest is nanoelectromagnetics, which covers electromagnetic wave theory and electromagnetic processes in quasione- and zero-dimensional nanostructures in condensed matter and nanocomposites with the focus on nanocarbon. He participates in a number of international research projects and is 
coordinator of EU FP7 project FP7-226529 BY-NANOERA.

Elena N. Golubeva received her MS degree in biophysics from Belarus State University, Minsk, Belarus, in 2011, where she is currently working toward the $\mathrm{PhD}$ degree. She is also a senior lecturer at the department of higher mathematics and mathematical physics, Belarus State University. Her current research interests include usage of Raman spectroscopy for investigation of interaction of single- and multiwall carbon nanotubes with living cells including cancer cells and cells of the human immune system.

Gintaras Valusis received his Diploma degree from the Faculty of Physics, Vilnius University, Vilnius, Lithuania, in 1985, where he received the PhD degree in 1992 . He was a postdoctoral researcher at the Institute of Applied Photo Physics, Dresden University of Technology during 1995 to 1996. In 2000, he was an Alexander von Humboldt Fellow at the Physics Institute, J.W. Goethe University, Frankfurt am Main, Germany. Currently, he is the director for development in the Center for Physical Science and Technology, and an professor in the Semiconductor Physics Department, Physics Faculty, Vilnius University.

Tommi Kaplas received his BE degree in Lahti Polytechnics (Finland) in 2005. After finishing his studies in Lahti Polytechnics, he began his studies in the University of Joensuu graduating with an MSc in physics in 2009. In 2010, he started his PhD studies in the University of Eastern Finland (UEF) graduating in 2013. After graduating he has been working as a post-doc in the UEF working in the field of carbon-based materials, synthesis and characterization.

Nicolas Reckinger received the M.S. degree in Electrical Engineering and the M.Sc. degree in Physics in 2000 and 2002, respectively, from the Universit catholique de Louvain, Louvain-la- 
Neuve, Belgium. At the same university, he pursued a Ph.D. degree in electrical engineering between 2008 and 2011. Since 2012, he is a postdoctoral researcher at the University of Namur, Belgium. His research interests include the fabrication and the characterization (both physical and electrical) of Si- and 2D-materials-based nanodevices, as well as the synthesis of 2D materials by chemical vapor deposition.

Michael Lobet received a M.S. degree (2011) and a Ph.D. (2016) in physical sciences from the University of Namur, Belgium. He is also a teaching assistant at the Department of Physics of the same university since 2011. His research interests are centered on metamaterials and 2D materials for photonic applications, with a focus on perfect electromagnetic absorbers.

Philippe Lambin received a M.S. degree in physical engineering from the University of Lige, Belgium, in 1976, and a Ph.D. in physical sciences from the same university in 1981. After having spent one year in the IBM Research Center, San Jose, Clifornia, as a visiting scientist, he joined the University of Namur, Belgium, as a research associate of the Foundation for Scientic Research of Belgium. He became professor of this university in 1995 and full professor in 2004. He was successively a dean of the faculty of sciences in 20052008 and vice-rector of the University of Namur in 20082011. His research interests include theoretical physics of the condensed matter and nanomaterials (atomic structure, vibrational and electronic properties). 


\section{List of Figures}

1 Graphene grain patchwork for small $(20 \mu \mathrm{m})$ (a) and large $(400 \mu \mathrm{m})$ (b) graphene grains drawn from experiment and transposed to RCWA calculations to investigate the effects of grain boundaries on the electromagnetic properties of a graphene/PMMA multilayer. The blue lines, $5.5 \mathrm{~nm}$ thick, have a dielectric permittivity of 1 , the rest has the permittivity of graphene. (c) Absorbance $(A)$, reflectance $(R)$ and transmittance $(T)$ of graphene/PMMA sandwich structures composed of $N$ graphene layers in case of graphene made of small (red lines) and large (blue lines) grains.

2 SEM images of graphene hexagonal domains corresponding to $20 \mu \mathrm{m}$-GRA (a) and $400 \mu \mathrm{m}$-GRA (c) samples, when the synthesis was stopped before graphene covered the whole copper substrate. (b), (d) optical images of graphene samples after chemical treatment obtained according to technique proposed in Ref. 27 . The disks drawn with a thin dotted line in (b) and (d) indicate the graphene domains in case of small and large grain size samples, respectively

3 Raman maps of the intensity ratio of D- and G-bands over a $40 \times 40 \mu \mathrm{m}$ area of $20 \mu \mathrm{m}$-GRA (a) and $400 \mu \mathrm{m}-\mathrm{GRA}$ (d), respectively. Raman maps of the intensity ratio of 2D- and G-bands over a $40 \times 40 \mu \mathrm{m}$ area of $20 \mu \mathrm{m}$-GRA (b) and 400 $\mu$ m-GRA (e), respectively. (c), (f) Raman spectra corresponding to the different regions marked with dots in (b) and (e). Spectra labelled 1 in (c) and 3 in (f) are typical of graphene monolayer. All spectra were normalized on the G band peak amplitude 
4 (a) Frequency dependence of $S_{11}$ and $S_{21}$ for graphene/polymer heterostructures on silica substrate consisting graphene with small and large grain size. Measured $S_{11}$ and $S_{21}$ for pure silica substrate (0.5 mm thickness) are shown inset. (b) The average SE of graphene/polymer heterostructures as function of the number of graphene layers at $30 \mathrm{GHz}$. 